\title{
Frontières
}

\section{Hagakuré et jihad}

\section{Double sens d'une mort volontaire obligatoire}

\section{Éric Volant}

Volume 15, numéro 2, printemps 2003

Guerre, mort amère

URI : https://id.erudit.org/iderudit/1073814ar

DOI : https://doi.org/10.7202/1073814ar

Aller au sommaire du numéro

\section{Éditeur(s)}

Université du Québec à Montréal

ISSN

1180-3479 (imprimé)

1916-0976 (numérique)

Découvrir la revue

Citer cet article

Volant, É. (2003). Hagakuré et jihad : double sens d’une mort volontaire obligatoire. Frontières, 15(2), 17-20. https://doi.org/10.7202/1073814ar
Résumé de l'article

Notre propos est de mettre en parallèle la mort volontaire des militaires japonais de la guerre du Pacifique et celle des militants islamistes du jihad en essayant de saisir de l'intérieur le sens de leur action mortifère. Nous présenterons d'abord le concept de mort volontaire altruiste et oblative à l'aide des typologies du suicide selon Émile Durkheim et Jean Baechler. Puis, nous confronterons ce concept aux attaques suicides des kamikazes japonais et aux attentats suicides des islamistes. Enfin, nous examinerons le bien-fondé de l'appellation kamikaze pour désigner ces deux types d'opérations, accomplies dans un contexte culturel et politique très différent.
Ce document est protégé par la loi sur le droit d'auteur. L'utilisation des services d'Érudit (y compris la reproduction) est assujettie à sa politique d'utilisation que vous pouvez consulter en ligne.

https://apropos.erudit.org/fr/usagers/politique-dutilisation/ 


\section{Résumé}

Notre propos est de mettre en parallèle la mort volontaire des militaires japonais de la guerre du Pacifique et celle des militants islamistes du jihad en essayant de saisir de l'intérieur le sens de leur action mortifère. Nous présenterons d'abord le concept de mort volontaire altruiste et oblative à l'aide des typologies du suicide selon Émile Durkheim et Jean Baechler. Puis, nous confronterons ce concept aux attaques suicides des kamikazes japonais et aux attentats suicides des islamistes. Enfin, nous examinerons le bien-fondé de l'appellation kamikaze pour désigner ces deux types d'opérations, accomplies dans un contexte culturel et politique très différent.

Mots clés : mort volontaire - disparu dépouille.

\section{Abstract}

The idea is to place, side by side, the voluntary death of the Japanese militia during the war in the Pacific with those of the Islamist jihad in order to grasp, from within, the meaning of this death act. We will first present the concept of the altruistic, selfless voluntary death with the use of typologies of suicide by Emile Durkheim and Jean Baechler. Then, we will confront the concept with the suicide attacks of the Japanese kamikaze and the suicide attacks of the Islamists. Finally, we will examine the pertinence of the term kamikaze to designate these two different operations, accomplished in extremely different cultural and political contexts.

Key words : voluntary death - missing human remains.

\section{HAGAKURÉ ET JIHAD DOUBLE SENS D'UNE MORT VOLONTAIRE OBLIGATOIRE}

\section{Éric Volant, Ph.D. professeur retraité, \\ Département des sciences religieuses, UQÀM.}

Étonnant, le tour imprévisible que peut prendre l'usage d'un nom! Kamikaze ${ }^{1}$, qui signifie « vent des dieux », est le nom attribué aux unités spéciales de la marine et de l'armée de l'air du Japon, formées à opérer des attaques suicides au cours des dix derniers mois de la guerre du Pacifique en 1944-1945. Effectivement, dans la matinée du 25 octobre 1944, sur les cinq appareils de l'escadrille Shikishima, quatre réussirent à percuter leur cible et à couler le porteavions Saint-Lô de l'armée américaine. En tout, 2198 jeunes pilotes, appelés Kamikazes, s'abîmaient avec leur avion en détruisant 34 navires américains et en causant des dommages à 288 autres. Près de soixante ans plus tard, dans la matinée du 11 septembre 2001, deux avions, détournés et pilotés par des militants islamistes, fonçaient, avec leur équipage et leurs passagers pris en otage, dans les tours jumelles du World Trade Center à New York et un troisième appareil frappait le Pentagone à Washington, causant la mort d'environ 3000 personnes. Les auteurs de ces attentats suicides sont, eux aussi, appelés Kamikazes, tout comme les Palestiniens soutenus par le Hamas qui, en faisant sauter les explosifs qu'ils portent sur eux, se tuent en semant la mort sur le sol d'Israël.

Notre propos est de mettre en parallèle la mort volontaire des militaires japonais de la guerre du Pacifique et celle des mili- tants islamistes du jihad ou de la lutte armée accomplie sur le sol américain. Dans la mesure du possible, nous essayerons de saisir de l'intérieur le sens de leur action mortifère et d'examiner la légitimité de l'utilisation du même nom pour désigner deux opérations anti-américaines, éloignées dans le temps et dans l'espace, accomplies dans des contextes politiques et culturels très distincts. Dans un premier temps, nous présenterons le concept de mort volontaire altruiste et oblative à l'aide des typologies du suicide selon Émile Durkheim et Jean Baechler. Dans un deuxième temps, nous confronterons ce concept à la mort volontaire des kamikazes japonais. Dans un troisième temps, nous étudierons, grâce au même concept, les caractéristiques des attentats suicides des islamistes et la pertinence de l'appellation kamikaze pour désigner cette opération ${ }^{2}$.

\section{SUICIDE ALTRUISTE ET OBLATIF}

Lorsque l'homme est détaché de la société, il se tue facilement, mais il se tue aussi lorsqu'il y est trop fortement intégré. C'est Durkheim (1981, p. 232-263) qui fait ce constat en appelant le geste du premier un suicide égoïste et celui du second, un suicide altruiste. Une individuation excessive peut conduire au suicide, tandis qu'une individuation insuffisante peut produire le même effet. Autrement dit, la tendance, à ne vivre que pour soi et la tendance qui n'accorde que peu de place à la personnalité individuelle dans la vie collective sont, toutes deux, susceptibles d'engendrer la crise suicidaire. 
Le terme altruisme exprime assez bien l'état où le moi ne s'appartient pas, mais se confond avec "autre chose que lui-même ». L'individu aspire à se dépouiller de son être personnel pour s'abîmer dans «cette autre chose» qu'il considère comme lui étant supérieure. C'est en elle et en elle seulement qu'il croit exister; c'est avec elle qu'il tend à se confondre. Le pôle de sa conduite est donc situé en dehors de lui, c'est-à-dire dans son groupe d'appartenance où la cohésion est massive et contraignante. Dans certains cas, la mort volontaire se présente alors non pas comme un droit dont on se réclame, mais comme un devoir qui s'impose. Absorbé dans la mouvance d'une collectivité qui se défend ou qui s'affirme, l'individu ne peut se soustraire à une mort qu'il estime indispensable à la cause commune. Durkheim donne le nom de suicide altruiste obligatoire au type ainsi constitué.

Baechler (1975, p. 221-237) construit sa typologie du suicide à l'aide de la méthode de l'« idéal-type » dont Max Weber s'est servi pour découvrir de l'intérieur le sens d'une action humaine (1965, p. 181). Il élabore quatre types de suicide qu'il divise en onze sous-types. Dans le cadre d'une étude des opérations kamikazes, le troisième type nous intéresse plus particulièrement. C'est celui du suicide oblatif ${ }^{3}$ que Baechler ne définit pas, mais qui nous semble très proche du suicide altruiste selon Durkheim et où il distingue deux soustypes. Le suicide sacrifice est «le fait d'attenter à sa vie pour sauver ou atteindre une valeur jugée supérieure à la vie personnelle », tandis que le suicide passage est «le fait d'attenter à sa vie pour accéder à un état considéré par le sujet comme infiniment plus délectable».

La personne qui accomplit un suicide sacrifice a préalablement assimilé une valeur qui lui est offerte par le groupe auquel elle appartient. Les valeurs varient selon les sociétés et, par conséquent, les sacrifices accomplis au nom d'une valeur collective sont propres à une société particulière. Pour cette raison, il est difficile sinon impossible de comprendre intimement une mort volontaire sacrificielle accomplie par des membres d'une autre société en vertu d'une valeur qui nous est étrangère ou peu promue dans notre communauté. Par le suicide passage, on espère atteindre un état supérieur ou entrer dans un monde, qualitativement différent de celui du présent.

Les attaques des kamikazes de la guerre du Pacifique des années 1940 et les attentats des islamistes radicaux à l'aube du XXI ${ }^{\mathrm{e}}$ siècle sont des suicides altruistes ou oblatifs par lesquels des jeunes, en vertu de certaines valeurs collectives, sacrifient leur vie au bénéfice de leur communauté d'appartenance. Mais il faut spécifier le contexte

historique, les valeurs et les traditions dont les auteurs se réclament, l'idéologie qui les porte et la cause qu'ils veulent servir.

\section{LE VENT DES DIEUX}

En 1994, l'armée japonaise se flatta de découvrir l'arme absolue et immatérielle, recette d'invincibilité, puisée dans les traditions lointaines et faisant appel à l'ardeur juvénile : la mort volontaire (Pinguet, 1984, p. 252-259 ; Baechler, 1975, p. 559-566). Le 19 octobre 1944, le vice-amiral Onishi, commandant des forces aéronavales des

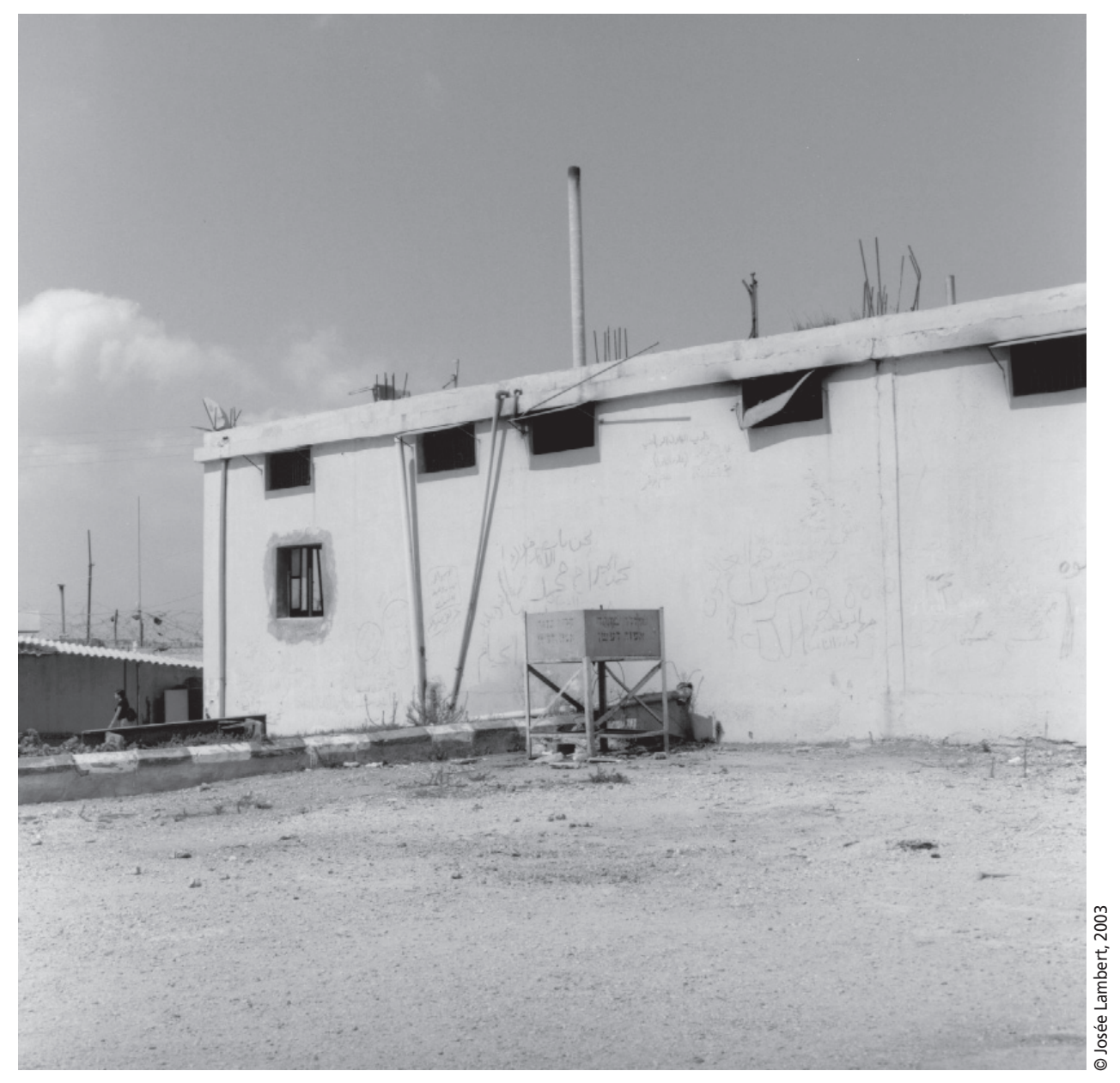

Là, les sueurs glacées de la peur suintent à la surface de la chaux.

Philippines, s'adressa aux pilotes assemblés à la base de Mabalacat. Les Américains venaient de réussir un débarquement et se préparaient à un combat naval décisif. Les stratégies de défense classiques étaient insuffisantes. Un espoir était permis si des chasseurs, chargés d'explosifs, allaient s'écraser sur les porte-avions ennemis. Le commandant faisait appel à des volontaires pour accomplir cette mission. Au début, on ne retenait que les sujets d'élite. Quelques mois plus tard, on sollicita des volontaires. Les commandants des bases aériennes rassemblaient des élèves officiers, évoquaient la nécessité du sacrifice pour l'honneur de la nation et demandaient une réponse pour le lendemain soir. De nombreux jeunes signaient leur engagement et commençaient leur entraînement. La plupart venaient des universités, surtout des disciplines juridiques et littéraires. Ils se jetaient dans le vide, heureux et fiers de mourir pour l'empereur et l'honneur du Japon.

Leur mort librement consentie était lucide et clairvoyante. Leur sacrifice volontaire avait le caractère de rationalité instrumentale et s'inscrivait à la rubrique des techniques militaires efficaces. Décidée par un État-major militaire, leur action avait pour cible les appareils militaires ennemis. Les jeunes kamikazes ne cherchaient aucune récompense ni dans cette vie ni dans une autre. Ils se conformaient aux arrêts du destin, comme l'un d'eux écrivait :

Je voudrais consacrer toutes mes forces à vivre ma vie d'homme en me conformant aux arrêts du destin. Voilà mon état d'esprit. [...]

Fuir cette voie qui nous a été tracée en recourant à des arguties est lâche [...] Je suis convaincu que c'est en consacrant toutes nos 
forces à la tâche qui nous incombe à chacun, que nous ferons progresser

l'Histoire du monde. Je veux vivre comme un homme parmi d'autres, jusqu'au bout humainement, sans lâcheté.

Ce témoignage, mises à part certaines expressions stéréotypées que l'on retrouve également dans les lettres de ses compagnons, révèle la représentation japonaise de la mort qui «a l'éclat étrange vif et frais du ciel bleu entre les nuages. » (Mishima, 1985, p. 90). Le Japon peut s'appuyer sur une longue tradition de la mort volontaire dans l'éthique samouraï. Le Hagakuré, grand classique de cette éthique du guerrier, contient les enseignements du samouraï devenu prêtre Jôchô Yamamoto (1659-1719) recueillis par écrit par son disciple Tsuramoto Thashino. Mishima a réinterprété le Hagakuré en fonction des temps modernes. Il estime que "l'esprit dans lequel ces jeunes hommes se précipitaient vers une mort certaine pour le salut de leur patrie est ce que la longue histoire du Japon offre de plus proche du clair idéal d'action et de mort proposé par le Hagakuré » (Mishima, 1985, p. 91). Même si c'est sous la contrainte qu'ils furent groupés en unités d'assaut et envoyés à une mort certaine, ils le firent de leur plein gré. D'après Mishima, il n'y a pas lieu de distinguer, dans ce contexte, entre la mort délibérément choisie et la mort imposée. À ce propos, l'écrivain cite le Hagakuré : «Je découvris que la Voie du samouraï, c'est la mort. Si tu es tenu de choisir entre la mort et la vie, choisis sans hésiter la mort» (Livre I, dans Mishima, 1985, p. 89).

\section{L'HEURE DE LA VINDICTE DE DIEU}

Les pilotes des avions suicides contre le World Trade Center et d'autres cibles américaines sont des jeunes musulmans provenant pour la plupart de l'Arabie Saoudite et recrutés dans les mosquées européennes. Sans passé religieux ni politique, ils se sont reconvertis à l'islam et se sont engagés dans le radicalisme politique. Ils ont été entraînés dans les camps établis en Afghanistan par le jihad afghan dont la mission de lutte armée est associée à un rigorisme religieux extrême. L'opération suicide à New York et à Washington fut décidée durant une période d'entraînement du réseau d'al-Qaïda (Diou et al., 2002, p. 417). Ben Laden avait commencé, dès la fin des années 1980, à constituer un fichier informatique, appelé en arabe al-Qaïda ou «base de données ». Il y répertoriait tous les volontaires qui se situaient dans la mouvance $d u$ jihad et transitaient par les camps de formation. Cette liste a été le point de départ d'un réseau clandestin assez nébuleux et plutôt décentralisé, réparti en

cellules opérant de façon indépendante, que nous connaissons sous le nom d'al-Qaïda (Kepel, 2000, p. 469-470) et dont Ben Laden est le chef présumé.

L'imaginaire commun des groupes d'islamistes radicaux se construit autour du jihad (Milot, 1975, p. 75-76; Grignard, 2002 ; Nasr, 1991, p. 145). Au départ, ce mot arabe signifie «faire un effort pour se maintenir sur le sentier de Dieu ». Aux premiers temps de l'islam, cet effort a pu prendre des allures militaires, destiné contre les incroyants non pas pour les exterminer, mais pour les délivrer de leur ignorance et instaurer parmi eux la loi de Dieu. Mais, très tôt, le jihad devint une lutte intérieure que tout musulman doit mener contre le mal destructeur qu'il porte dans son âme afin d'emprunter la voie tracée par Dieu. Dans la théologie classique, la lutte armée est appelée "petit jihad», tandis que le " grand jihad » désigne le combat intérieur. De nos jours, l'islamisme radical préfère l'activisme politique à la spiritualité intérieure fondée sur la foi prêchée dans la communauté des croyants. Il vise la reconquête $\mathrm{du}$ pouvoir politique par la restauration de

\section{IL EST DIFFICILE SINON IMPOSSIBLE DE COMPRENDRE INTIMEMENT}

UNE MORT VOLONTAIRE SACRIFICIELLE ACCOMPLIE

PAR DES MEMBRES D'UNE AUTRE SOCIÉTÉ EN VERTU D'UNE VALEUR QUI NOUS EST ÉTRANGÈRE.

la religion islamique dans son intégralité et l'établissement d'un état islamique fort, à visée internationaliste, qui gouvernerait selon la Charia ou loi de Dieu et réparerait l'injustice subie par les musulmans à travers le monde (Blanc, 2002, p. 21).

Dans le cadre de ses études d'ingénieur à l'université du roi Abd-al-Aziz de Djedda, le jeune Oussama Ben Laden aurait été l'élève de Abdallah Azzam (1941-1989), professeur d'études islamiques et membre des Frères musulmans ${ }^{4}$. En 1982 à Peshawar sur la frontière du Pakistan et de l'Afghanistan, il mettra sur pied une maison d'hôtes pour des jihadistes internationaux avec Azzam, devenu le héraut et la figure de proue du jihad afghan. Azzam estimait que la défense de la terre des musulmans était le plus important devoir de chacun. Chaque musulman a l'obligation individuelle de participer au jihad moralement et financièrement et, s'il en est capable, par les armes (Kepel, 2000, p. 217-224). Pour Azzam, le jihad fut surtout une guerre défensive, mais Ben Laden en fera une guerre offensive contre les Américains. des croisés, les militants internationalistes demandent une fatwa, un édit qui les assure que leur action recevra la bénédiction d'Allah.

Les auteurs des attentats suicides sur New York et Washington ont pu lire, la veille de leur exploit, un vade-mecum, qu'on aurait trouvé après les événements dans les bagages laissés dans l'aéroport de Washington. Ce guide, qui a pour fonction de conjurer la peur et de leur donner espoir, présente leur course meurtrière vers l'abîme comme un passage obligé vers le ciel :

Voici le moment du Jugement. Tu vas ressentir un calme total. Car le temps qui te sépare de ton mariage au ciel est très court. Aiguise ton couteau pour ne pas faire souffrir l'animal que tu abats. La peur est une immense adoration. Sois heureux, optimiste et calme, car tu t'apprêtes à effectuer un acte qui plaît à Dieu (Venner, 2002).

L'engagement dans ce type de groupes militants implique nécessairement une rupture biographique qui passe par une renonciation à une identité antérieure, 
l'intériorisation de règles de comportement codifiées, l'entrée dans la clandestinité, la familiarité avec la mort et la logique de l'accès à la vraie vie (Sommier, 2000, p. 115). Olivier Roy trace le profil type des militants islamistes internationalistes (2002, p. 185204). Ce sont des musulmans transnationaux qui ont quitté leur pays d'origine et ont vécu dans plusieurs pays ayant parfois acquis la nationalité française, britannique, canadienne ou américaine. Issus des classes moyennes ou des quartiers populaires sensibles, ils ont fait des études de qualité dans le domaine de la technologie moderne et ont vécu une jeunesse à l'occidentale. Leur conversion à l'islam s'est effectuée en Occident à la suite de rencontres dans une mosquée radicale et, de ce fait, coïncide avec leur passage au radicalisme politique. Ils ont rompu avec leur famille, leur pays d'origine et leur pays d'accueil et ont rejoint une fraternité internationale.

Avant les événements du 11 septembre 2001, Fahrad Khosrokovar avait déjà développé le concept de "martyropathie» pour désigner l'esprit qui habitait les islamistes radicaux en Iran.

Le martyropathe meurt par incapacité de vivre. [...] Il inaugure un type inattendu de mort [...]

Cri de protestation, de désespoir, la martyropathie est l'indice de cette subjectivité nouvelle qui [...] dénonce, par la mort, le scandale d'une vie vouée au non-sens [...], à la déshérence (situation de déshérité) et à l'inaptitude à s'assumer [...] Plus généralement, le martyre révolutionnaire est, dans son essence, d'abord une provocation [...] devant l'ordre mondial. À l'incertitude et à l'insécurité généralisées dans un monde de rêve qui s'écroule, il oppose la nouvelle certitude, sa certitudo mortis

(Khosrokovar, 1995, p. 48).

La fascination de certains jeunes adultes pour la mort naît du désir de s'affirmer et d'accomplir le projet commun de liberté et d'égalité. Se rendant compte que ce double désir d'autonomie personnelle et collective du monde arabe ne pourra jamais se réaliser durant leur vie, ils choisissent avec détermination la mort. Ils optent pour une rupture radicale avec un monde qu'ils ne parviennent pas à changer.

\section{SAUT DANS LE VIDE ET VOYAGE PARADISIAQUE}

La mort volontaire des militaires japonais et des militants islamistes est un suicide altruiste et oblatif, un consentement libre au sacrifice de leur vie pour servir ou atteindre «autre chose»(une altérité), étroitement liée à leur communauté d'appartenance. L'opération kamikaze, accomplie dans le cadre d'une guerre dite classique entre deux ennemis, est un suicide altruiste au service d'une cause nationale et inspirée par une longue tradition de mort volontaire selon l'éthique du samouraï. Elle est une stratégie militaire efficace ayant une cible précise, les équipements militaires américains et les soldats ennemis qui s'y trouvent. Elle s'accomplit sur fond d'une mystique tragico-héroïque, inculquée par l'état-major de l'armée. Le guerrier combattant, fier de servir la cause de l'empereur, personnage sacré, et l'honneur de sa nation, fait le saut dans le vide et assume son destin en s'abîmant dans la mort. Lui seul mérite le nom de kamikaze, qui appartient à la tradition et à la culture japonaises.

Les attentats suicides ont été exécutés par des militants islamistes dans la conjoncture du jihad, lutte armée transnationale où le religieux et le politique sont intimement liés. Le croyant se soumet à la volonté de Dieu, prêchée par des imams fondamentalistes, et accomplit la vindicte divine en ciblant, avec précision, des symboles de l'hégémonie américaine entraînant dans la mort une population civile «imprécise ${ }^{5}$, jugée non pas innocente, mais vivant dans l'ignorance et donc complice par sa participation au système capitaliste occidental, estimé foncièrement injuste et inéquitable. Son but est le renversement de l'hégémonie occidentale, la restauration de la foi en Dieu et la mondialisation de l'islam dans sa pureté intégrale. Une guerre totale! La destinée ultime du croyant combattant est la récompense de Dieu à qui il a voulu plaire par son action mortifère: le passage au paradis et la jouissance de la vie éternelle.

\section{Bibliographie}

AL-BERRY, K. (2002). La terre est plus belle que le paradis, Paris, J.C. Lattès.

BAECHLER, J. (1975). Les suicides, Paris, Calmann-Lévy.

BLANC, F. (2001). Ben Laden et l'Amérique, Paris, Bayard.

DIOU, K. A. et al. (2002). "Quaïda », dans Dictionnaire mondial de l'Islamisme, Paris, Plon, p. 417.

DURKHEIM, É. (1981). Le suicide. Étude de sociologie, Paris, Quadrige / PUF.

GRIGNARD, A. (2002). "Brève genèse de l'islamisme radical», dans Gérard CHALIAND, L'arme du terrorisme, Paris, L'Audibert.

KEPEL, G. (2000). Jihad. Expansion et déclin de l'islamisme, Paris, Gallimard.
KHOSROKOVAR, F. (1995). L'islamisme et la mort. Le martyr révolutionnaire en Iran, Paris, L'Harmattan, "Comprendre le Moyen Orient ».

MILOT, J.-R. (1975). L'Islam et les musulmans, Montréal, Fides.

MISHIMA, Yukio (1985). Le Japon moderne et l'éthique samouraï. La voie $d u$ Hagakuré, Paris, Gallimard.

NASR, F.H. (1991). Islam. Perspectives et réalités, Paris, Buchet-Chastel.

PINGUET, M. (1984). La mort volontaire au Japon, Paris, Gallimard, "Bibliothèque des histoires".

ROY, O. (2002). Islam mondialisé, Paris, Seuil, «La couleur des idées».

SOMMIER, I. (2000). Le terrorisme, Paris, Flammarion, «Dominos».

VENNER, D. (2002). Histoire du terrorisme, Paris, Pygmalion, Gérard Watelet.

VOLANT, É. (2002). "Une journée dans la vie d'un kamikaze », Nouveau dialogue, $\mathrm{n}^{\circ} 140$.

VOLANT, É. (2001). «Kamikaze», dans Dictionnaire des suicides, Montréal, Liber.

WEBER, M. (1965). Essai sur la théorie de la science, Paris, Plon.

\section{Notes}

1. L'expression japonaise Kamikaze Tokubetsu Kôgekitai signifie forces d'attaque spéciales du vent des dieux, en abrégé Tokkôtai ou forces spéciales, couramment nommées à l'étranger kamikaze ou vent des dieux (Pinguet, 1984, p. 354).

2. Dans le présent article, nous nous limiterons au sens du geste, à sa signification politique et religieuse. Nous avons traité de l'appréciation éthique de ces deux opérations dans le Dictionnaire des suicides, 2001, article « Kamikaze », p. 179-180 et dans «Une journée dans la vie d'un kamikaze », 2002, p. 9-10 et 16 , où nous avons mis en évidence le caractère aliénant d'un endoctrinement idéologique fait par des chefs à des jeunes, vulnérables dans leur idéalisme. La subjectivité des personnes, en préparant ou en accomplissant leur action suicidaire et meurtrière, ne nous est guère accessible.

3. «Oblatif» est défini par Le Petit Robert comme suit: "Qui s'offre à satisfaire les besoins d'autrui au détriment des siens propres.» C'est nous qui soulignons.

4. Association, fondée par Hassan-Al-Banna en 1927-1928 en Égypte, qui prôna l'islamisation des pratiques sociales et éducatives et fut assassiné en 1949, de manière non encore élucidée. Sayid Qotb (1906-1966) radicalisa le mouvement et se fit le défenseur de la violence politique contre la civilisation occidentale. Il fut assassiné durant le règne de Nasser.

5. Nous utilisons ici le terme "imprécise», parce que le nombre et la douleur des victimes ainsi que le deuil de leurs survivants pèsent peu dans la balance par rapport à l'objectif visé : la reconquête de l'hégémonie de l'islam. 